\title{
Institutional preparedness strategies for heart failure, durable left ventricular assist device, and heart transplant patients during the Coronavirus Disease 2019 (COVID-19) pandemic
}

Aditya Bansal, MD, ${ }^{\mathrm{a}}$ Daniel Goldstein, MD, ${ }^{\mathrm{b}}$ Sarah Schettle, PA-C ${ }^{\mathrm{c}}$ Stephen Pepitone, MBA, ${ }^{\mathrm{d}}$ Brian Lima, MD, ${ }^{\mathrm{e}}$ Duc T. Pham, MD, ${ }^{\mathrm{f}}$ Jennifer Cowger, MD, ${ }^{\mathrm{g}}$ Armin Schubert, MD, ${ }^{\mathrm{h}}$ and Sean P. Pinney, MD

During the Coronavirus Disease 2019 (COVID-19) pandemic, patients with heart failure still require care. During the acute phase of the pandemic, heart failure teams at 6 medical centers located in 4 infection hotspots-New York, New Orleans, Detroit, and Chicago—continued to care for patients with heart failure and patients supported by ventricular assist devices (VADs) and served as resource centers for small hospitals and VAD centers. These teams collaborated to coalesce their strategies for tackling the challenges of this pandemic to help other institutions improve preparedness and potentially reduce mortality and suffering.

Each hospital already had emergency preparedness plans at the institutional level and for patients with heart failure and VAD-supported patients that were developed before the pandemic. Despite some differences, many strategies were similar, and we have categorized these best practices for managing patients with heart failure during crisis situations as the 4Cs: Capacity, Cohort, Care, and Collaboration (Figure 1).

\section{CAPACITY}

Triage is essential during critical times with limited resources. ${ }^{1}$ Early identification of patients who could be discharged helped protect them from exposure to the virus, decompressed the hospital, and saved resources for the

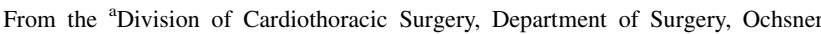
Clinic Foundation, New Orleans, La; ${ }^{\mathrm{b}}$ Department of Cardiothoracic Surgery, Montefiore Medical Center, New York, NY; ${ }^{\mathrm{c}}$ Division of Cardiovascular Surgery, Mayo Clinic, Rochester, Minn; ${ }^{\mathrm{d}}$ Patient Throughput, Ochsner Clinic Foundation,

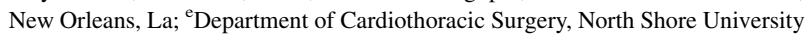
Hospital, Northwell Health, Manhasset, NY; ${ }^{\mathrm{f}}$ Department of Cardiothoracic Surgery, Northwestern University Feinberg School of Medicine, Chicago, Ill; ${ }^{\text {gDepart- }}$ ment of Cardiology and Heart Failure, Henry Ford Hospital, Detroit, Mich; ${ }^{\mathrm{h}}$ Department of Anesthesia and Critical Care Medicine, Ochsner Clinic Foundation, New Orleans, La; and ${ }^{\mathrm{i}}$ Department of Cardiology and Heart Failure, Mount Sinai Hospital, Icahn School of Medicine at Mount Sinai, New York, NY.

Received for publication May 4, 2020; revisions received July 30, 2020; accepted for publication Aug 8, 2020; available ahead of print Sept 2, 2020.

Address for reprints: Aditya Bansal, MD, Section of Cardiothoracic Surgery, Department of Surgery, Ochsner Clinic Foundation, 1514 Jefferson Hwy, New Orleans, LA 70121 (E-mail: adbansal@ochsner.org).

J Thorac Cardiovasc Surg 2021;162:131-5

$0022-5223 / \$ 36.00$

Copyright (c) 2020 by The American Association for Thoracic Surgery

https://doi.org/10.1016/j.jtcvs.2020.08.096
}

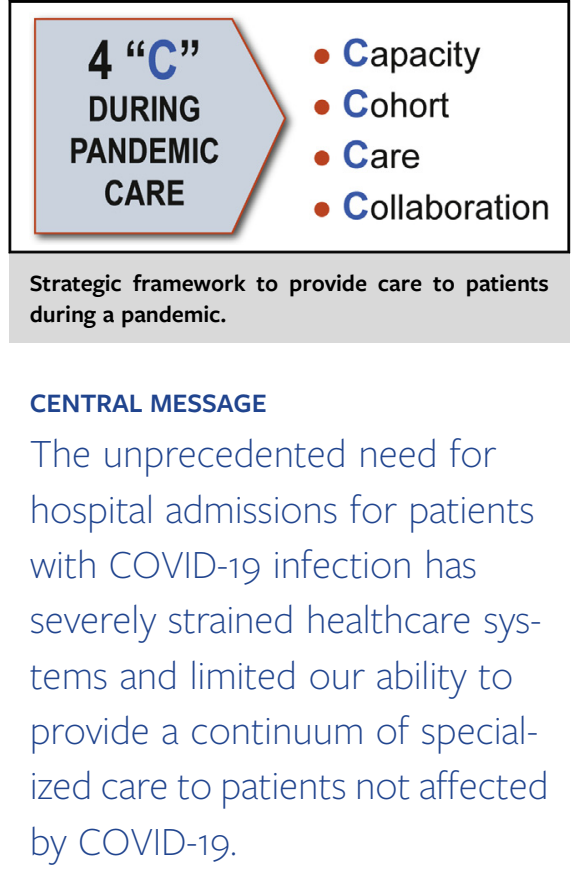

See Commentaries on page 136,137 , and 138 .

surge of patients with COVID-19. Using institutional and governmental predictive models to anticipate the surge and peak of the pandemic helped us schedule advanced surgical care for patients with heart failure..$^{2-5}$ Forecasting intensive care unit (ICU) and ventilatory support for patients with a new left ventricular assist device (LVAD) further assisted with planning. Some centers shifted care for recent transplant recipients to COVID-19-free facilities and placed transplant teams there to provide care. Most centers had severe bed, ventilator, personal protective equipment (PPE), and blood product constraints during the acute phase of the pandemic, so cardiac transplant was halted at most centers. Mechanical circulatory support (MCS) therapies were provided for patients with Interagency Registry for Mechanically Assisted Circulatory Support 1 and 2 profiles, cases in which delaying care would be imprudent. LVAD surgery is 


\section{Institutional Preparedness for Heart Failure Patients with COVID-19}

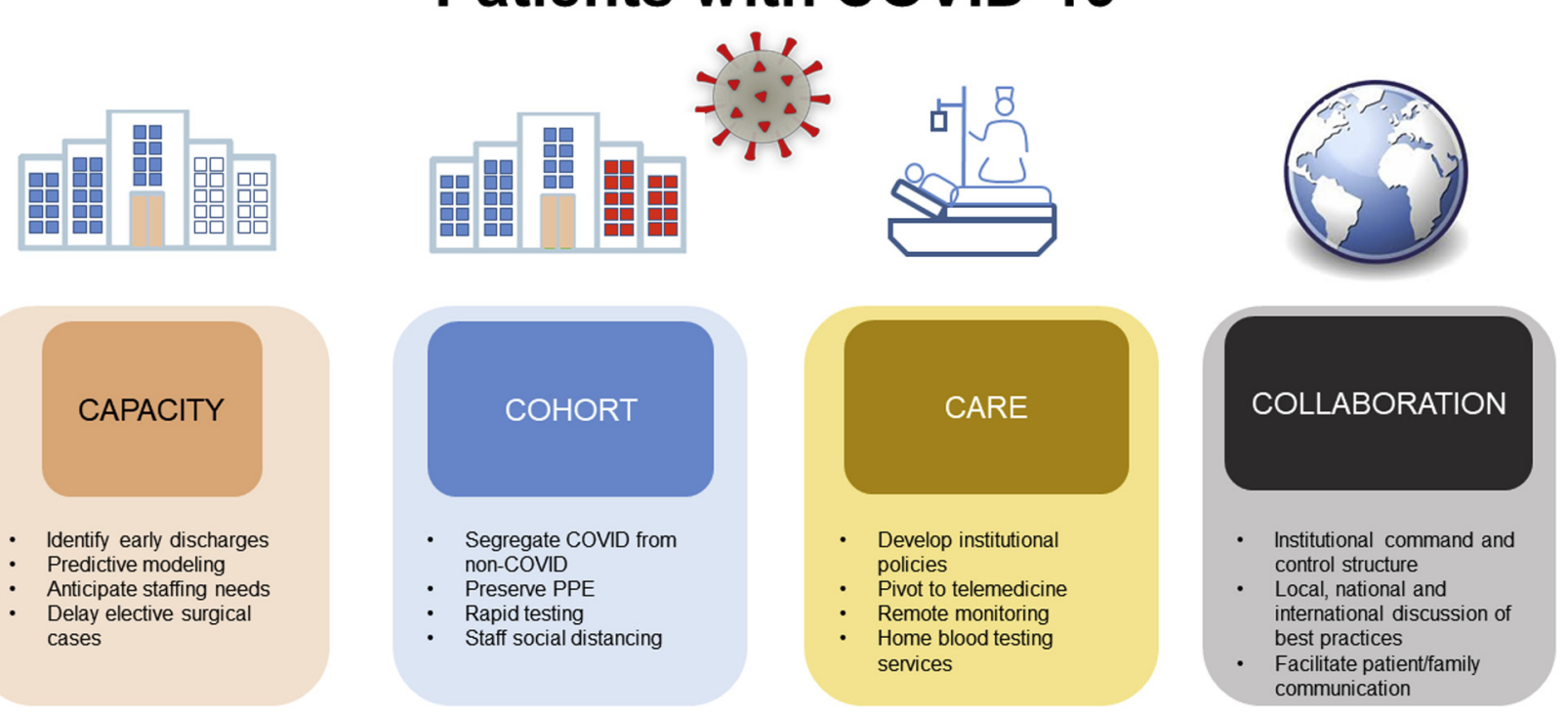

FIGURE 1. Schematic showing the $4 \mathrm{C}$ strategies for institutional preparedness with implementation steps. COVID-19, Coronavirus Disease 2019; PPE, personal protective equipment.

resource intensive and so is caring for critically ill patients with COVID-19. However, one should not risk having a patient die of heart disease to save someone dying of COVID19. Both patients deserve care even if they are resource intensive. Patients on the heart transplant list were notified about the temporary suspension of the program. These strategies quickly decompressed inpatient services and opened beds for patients with COVID-19. Appropriate staffing of the cardiac catheterization laboratory, endoscopy suites, and operating rooms also added beds. Critical care capacity should be addressed early. Targeted organizational effort should be devoted to make additional critical care resources available: space, equipment, and personnel. Some centers found it helpful to implement an internal resource center for redeploying staff and material.

\section{COHORT}

Grouping COVID-19-positive patients with heart failure in one part of the hospital/ICU is critical to prevent the spread to noninfected patients, ${ }^{6,7}$ allows for care by a minimal number of VAD-trained physicians and VADcertified nurses, and helps conserve PPE. Every patient being admitted or transferred should undergo COVID-19 testing. Patient under investigation units were created for patients with suspected COVID-19 awaiting test results. Treatment teams should use appropriate PPE and round on all COVID-19-negative patients before rounding on COVID-19-positive patients.

Organizations can limit workforce exposure by facilitating remote work. ${ }^{8}$ Support functions in many organizations-including finance, supply chain, communications, administration, office staff, medical informatics, and information technology — continued productive operations while working remotely. To maintain social distancing, most centers rotated staff in variable time blocks to reduce potential for cross-contamination.

Rapid expansion of care team capacity for creating patient cohorts is difficult because of the delays inherent in recruitment and sourcing of temporary workers. Just in time capacity expansion relies on quickly creating new team structures. At one center, critical care and hospitalist capacity was doubled (effectively adding 100 ICU beds) by assembling volunteer provider teams and redeploying other care team members. Assembling these teams was facilitated by (1) ensuring access to expertise in critical and hospital medicine care through a professional familiarity system; (2) distributing and ensuring on-demand access to a minimum level of training resources; and (3) limiting the discomfort factor.

A professional familiarity system can reduce stress and promote team cohesion. For example, surgical or neurointensivists can manage a tiered staffing system that includes surgical subspecialists and other procedurally oriented physicians. Anesthesiologists or interventional radiologists can bolster newly created ICU teams by taking responsibility for most procedural care (placement of endotracheal tubes, central lines, and arterial lines), thereby freeing intensivists for patient care issues.

Professional disruption and discomfort can be limited by redeploying providers in no more than 2 unfamiliar dimensions. For example, a volunteer ear, nose, and throat surgeon could be introduced into a critical care team ( 2 
unfamiliar dimensions: type of practice and disease) but in the ear, nose, and throat surgeon's hospital, preserving the geography dimension. Redeployment discomfort can be further reduced by staffing as robustly as possible to overcome unexpected outages from COVID-19 exposure and ensure manageable workloads during steep learning curves. Whole teams should be deployed to specific units to improve team functioning and learning. This tactic can also reduce exposure, especially in fully isolated units where PPE is worn throughout the shift; cohesive teams rapidly learn to keep each other safe. At many centers, these strategies resulted in an expansion of ICU capacity by $100 \%$ in a short period.

\section{CARE}

\section{Telemedicine and Remote Monitoring}

Providing ongoing care to vulnerable patients during a pandemic is critical to prevent the need for emergency hospital care that would place them at risk of exposure and strain emergency medical services. Exposure can be mitigated through telemedicine virtual visits. Most patients had their concerns and issues resolved via a virtual clinic visit within 24 hours of contact with the VAD center. For emergencies requiring immediate attention, patients were seen promptly and provided care. During the pandemic, the Centers for Medicare \& Medicaid Services facilitated increased provision of telehealth, expanding payment for visits to patients outside rural areas. ${ }^{9}$ Under the CARES Act, providers at federally qualified health centers and rural health clinics can offer and bill for virtual communication of 5 minutes or more with patients. ${ }^{10}$ Remote monitoring of patients with LVADs should include 5 dimensions: LVAD controller alarms, blood pressure, pacemaker analysis, coagulation values (international normalized ratio monitoring), and smart phone-transmitted findings (eg, driveline photos). ${ }^{11}$ Currently, this monitoring requires patients with LVADs to collect, review, and transmit data to the care team. Just as COVID-19 accelerated the acceptance of telemedicine visits, the hope is that the pandemic will accelerate the automation of remote VAD care, making it more real-time with less dependence on patient participation. Many centers expanded home telemanagement via patient-assisted wireless/cellular weight, blood pressure, and heart rate monitoring (through Alere [Waltham, Mass], Cordella [Endotronix Inc, Ill]). Despite limited and variable reimbursement, many personnel in centers thought this monitoring added a necessary level of patient oversight. Many centers encouraged and offered home international normalized ratio monitoring when insurance allowed. Heart transplant recipients who are being monitored for rejection with protocol endomyocardial biopsies may be considered for noninvasive molecular diagnostics instead. Some may be eligible for a home blood service that collects and transports the specimens. Patients more than 55 days from transplant and receiving less than $20 \mathrm{mg}$ of prednisone may be eligible for this service.

The CardioMEMS HF System (a Food and Drug Administration-approved heart failure monitor; Abbott, Abbott Park, Ill) has been shown to reduce heart failure hospital admissions by $58 \%$ per year. ${ }^{12}$ Many centers used this tool to adjust medications on the basis of a patient's pulmonary pressure and reduced hospital visits during the pandemic.

\section{Supply Chain Medications and Ventricular Assist Device Supplies}

During the pandemic, retail pharmacies can dispense 90 days of maintenance and lifesaving medications (immunosuppression) by using the disaster relief override provision in insurance policies, permitting an uninterrupted supply of medications to patients with heart failure and heart transplant recipients complying with shelter-at-home orders.

Patients who run out of supplies must be provided with an emergency supply through the nearest shared care site or from their implant center. Strong lines of communication should be maintained between home infusion therapy services and patients supported on home inotropes to prevent running out of inotropes.

\section{Ventricular Assist Device Coordinators and Social Workers}

Lack of physical contact and interaction can precipitate anxiety and depression. VAD coordinators and social workers can contact VAD-supported patients weekly to provide emotional support and to triage medical problems. For patients in the hospital, coordinators can help them connect with family members via mobile device technology during restricted visitation. These strategies proved extremely helpful in maintaining the psychologic well-being of patients and family members.

Patient and caregiver education about LVAD implantation is another challenge of the COVID-19 era. Although verbal and hands-on education traditionally occur in person in the hospital setting, instruction has been augmented or replaced with video conferencing supplemented with hospital-developed or industry-created video educational materials. Video conferencing allows caregivers to demonstrate basic MCS competency through teach-back and response to device alarm simulations. Videos demonstrating proper driveline care, equipment care and handling, and alarm recognition can also be used postpandemic for continued education. The primary caregiver can be loaned replica controllers and batteries to practice techniques at home and during virtual conferencing. Before discharge, the primary caregiver can demonstrate through hands-on skill assessment the knowledge gained from virtual conferencing and education videos. 


\section{Protocols}

Cross-coverage with different services and specialists is essential, and protocols will help standardize care. Standardized management protocols and sharing clinical practice guidelines can guide team members who do not regularly care for patients with heart failure. A 24-hour point-of-contact individual from the heart failure team is a critical resource for cross-covering physicians. Electronic medical record-based order sets can further guide treatment teams and help reduce errors.

\section{Transfer of Care}

VAD centers that have reached their admission capacity because of COVID-19 should plan to transfer patients with heart failure needing hospitalization to other regional VAD centers. Rapid transfer can be lifesaving for patients needing urgent therapies. Family members must be provided the contact information for the regional VAD center to prevent confusion and concern. At most centers, weekly multi-institutional conference calls with the transplant/ MCS programs-inclusive of MCS, ECMO, and critical care program directors-allowed for efficient patient comanagement, as well as discussion of center-specific bed and ECMO capacities.

\section{Care of Frontline Workers}

Decompression zones in ICUs can help reduce stress and recharge frontline providers. Provision of sufficient PPE and food is much appreciated. Daily rounding of mental health specialists in the ICUs and wards provides consistent and welcome access for healthcare providers dealing with psychological stressors. Virtual town hall meetings provide a mechanism to highlight and recognize individuals and their contributions. Administrative and medical leaders should be visible on hospital floors and other care areas to reassure patient-facing team members that leaders are concerned for their safety and ability to care for patients optimally.

\section{Ethics Regarding Resource-Constrained Care}

Convening a multidisciplinary group that applies ethical principles to decisions that must be made during a pandemic response relieves individual providers of the burden of making decisions in isolation. Organizations should review state statutes and regulations applicable to crisis response. At some centers, groups reviewed state statutory guidance from the $2010 \mathrm{H} 1 \mathrm{~N} 1$ influenza pandemic. ${ }^{13}$ By using the ethical principles from these state documents, guidance was developed for contingency and crisis standards of care. Contingency care is defined as the usual standard of care during which practice changes can be adopted that effectively deliver care with the same outcome. Crisis care refers to a radically different standard of care in which triage decisions must be made to govern deployment of severely compromised resources. An example of developing guidance according to ethical principles is basing decisions on assessment of probability of survival. Decision-making is independent of disease (eg, COVID-19) and is not constrained by the type of intervention contemplated, including advanced care options of VAD implantation or heart transplantation. Under such guidance, a transplant team would make care decisions based on estimated survival, risk of the intervention, and risk of delaying care. Many centers chose a nondiscriminatory ethical approach during the pandemic. Preexisting conditions or presence of an LVAD or heart transplant did not prioritize or discriminate patients regarding COVID-19 care.

\section{COLLABORATION}

\section{Chain of Command}

A chain of command chart is essential for identifying those who can rapidly address the needs of frontline providers. An institutional level COVID Command Center is critical for informing employees about strategies being implemented and for stopping disinformation and rumors. Another essential function of the command center is to build confidence among care teams that their requests for assistance are heard and mitigated.

\section{Use of Social Media}

Reverse engineering techniques that are shared via social media, such as the decontamination and safe reuse of N95 masks and placing LVAD patient system monitors outside of patients' rooms, sound helpful. However, before any social media-based recommendations are implemented, they must be fully vetted through institutional safety committees to ensure the protection of patients and employees.

\section{Mass Quarantine of Workforce}

Every effort should be made to prevent the spread of infection by aggressive testing and isolation of frontline workers. ${ }^{14}$ Essential personnel should be rotated to prevent complete team quarantine. However, in situations of mass quarantine, redeployment of physicians, nurses, Certified Registered Nurse Anesthetists, and advanced practice providers from different subspecialties can help fill gaps. Agency nurses can help staff new ICU beds.

\section{Communicating With Families of Severely Ill or Dying Patients}

The COVID-19 pandemic restricted hospital access of patients' families and loved ones. Everyone can relate to the fear of dying alone in the hospital. ${ }^{15}$ Social workers, VAD coordinators, and hospice and pastoral services staff can provide the means for patients and family members to say their final goodbyes and help support grieving families in times of personal loss. 


\section{Preparation for Reopening/Resurgence/New Spike}

Once cities and centers experienced a constant decline in new COVID-19 cases, planning for reopening was initiated and synchronized with state and city phased reopening guidelines for surgical cases. Patients on the heart transplant waiting list were informed of visitation restrictions to help them make informed decisions regarding being considered for transplant.

Most centers reopened their transplant programs when (1) ICU bed and ventilator capacity allowed; (2) PPE and staffing were sufficient; and (3) testing (COVID-19 and usual workup for pretransplant and pre-LVAD) was fast and efficient.

Once transplant programs reopen, coordination with the local organ procurement organization is critical, and efforts should be made for procurement and use of organs locally by in-state centers to increase donor use and minimize long-distance travel for out-of-state procurement teams.

Hospitals need to prepare for a possible increase of COVID-19-positive cases as social distancing norms are relaxed and phased reopening of society occurs, as witnessed in South Korea, Singapore, and Japan. In the downward phase of the infection curve, patients admitted to the hospital with COVID-19 infection should be consolidated to help free space for decontamination of ICU and hospital beds. This strategy will help institutions prepare for the increase in cases once elective and semi-urgent care are delivered again. Strict hospital visitor policies should be maintained until the incidence of infection in the community decreases or per the recommendation of governmental agencies. Rapid screening of outpatients, social distancing in waiting areas, and the use of PPE will help to minimize the risk of contagion.

\section{CONCLUSIONS}

Collaboration is critical during a global crisis. Cooperation with local, regional, and state officials and with other hospitals in the region will enhance resource use. ${ }^{16}$ At the institutional level, departmental silos need to be unsealed to provide care for critically sick patients across the multidisciplinary continuum. Collaboration with and learning from different centers will prove helpful in compiling our collective experiences. During times of travel restrictions, webinars can be used for rapid dissemination of information. Incorporating these strategies to optimize patient care can help institutions with preparedness planning and responsiveness to this pandemic.

\section{Conflict of Interest Statement}

The authors reported no conflicts of interest.

The Journal policy requires editors and reviewers to disclose conflicts of interest and to decline handling or reviewing manuscripts for which they may have a conflict of interest. The editors and reviewers of this article have no conflicts of interest.

\section{References}

1. Ferraresi M. A Coronavirus cautionary tale from Italy: don't do what we did. The Boston Globe. Available at: www.bostonglobe.com/2020/03/13/opinion/corona virus-cautionary-tale-italy-dont-do-what-we-did/. Accessed April 15, 2020.

2. Wynants L, Van Calster B, Collins GS, Riley RD, Heinze G, Schuit E, et al. Prediction models for diagnosis and prognosis of covid-19 infection: systematic review and critical appraisal. BMJ. 2020;369:m1328

3. Dong E, Du H, Gardner L. An interactive web-based dashboard to track COVID19 in real time. Lancet Infect Dis. 2020;20:533-4.

4. Grasselli G, Pesenti A, Cecconi M. Critical care utilization for the COVID-19 outbreak in Lombardy, Italy: early experience and forecast during an emergency response. JAMA. 2020;323:1545-6.

5. Xie J, Tong Z, Guan X, Du B, Qiu H, Slutsky AS. Critical care crisis and some recommendations during the COVID-19 epidemic in China. Intensive Care Med. 2020;46:837-40.

6. Carenzo L, Costantini E, Greco M, Barra FL, Rendiniello V, Mainetti M, et al. Hospital surge capacity in a tertiary emergency referral centre during the COVID-19 outbreak in Italy. Anaesthesia. 2020;75:928-34.

7. Carinci F. Covid-19: preparedness, decentralisation, and the hunt for patient zero. BMJ. 2020;368:bmj.m799.

8. Stopping COVID-19. Available at: www.mitre.org/publications/technical-pape rs/white-paper-stopping-covid-19-short-term-actions-for-long-term-impact. Accessed June 9, 2020.

9. List of Telehealth Services: Covered Telehealth Services for PHE for the COVID-19 pandemic, effective March 1, 2020 (ZIP). Centers for Medicare \& Medicaid Services. Available at: www.cms.gov/Medicare/Medicare-GeneralInformation/Telehealth/Telehealth-Codes. Accessed April 21, 2020.

10. Federally Qualified Health Centers (FQHC) Center: COVID-19 Public Health Emergency (PHE) - Updates for FQHCs. Centers for Medicare \& Medicaid Services. Available at: www.cms.gov/Center/Provider-Type/Federally-QualifiedHealth-Centers-FQHC-Center. Accessed April 21, 2020.

11. Reiss N, Schmidt T, Boeckelmann M, Schulte-Eistrup S, Hoffmann JD Feldmann C, et al. Telemonitoring of left-ventricular assist device patientscurrent status and future challenges. J Thorac Dis. 2018;10(Suppl 15):S1794-801.

12. Abraham WT, Adamson PB, Bourge RC, Aaron MF, Costanzo MR, Stevenson LW, et al. Wireless pulmonary artery haemodynamic monitoring in chronic heart failure: a randomised controlled trial. Lancet. 2011;377:658-66.

13. Louisiana Crisis Standards of Care: A guideline for Louisiana's Acute Care Hospitals, V1.2, September 2011. Available at: cdn.ymaws.com/www.lhaonline.org/ resource/resmgr/imported/Crisis \% 20Standards \% 20Public $\%$ 20Document $\% 20$ Acute.pdf. Accessed June 9, 2020.

14. COVID-19: protecting health-care workers. Lancet. 2020;395:922

15. Wakam GK, Montgomery JR, Biesterveld BE, Brown CS. Not dying alone - modern compassionate care in the covid-19 pandemic. N Engl J Med. 2020;382:e88.

16. Singer AJ, Morley EJ, Henry MC. Staying ahead of the wave. $N$ Engl J Med. 2020;382:e44.

Key Words: pandemic, COVID-19, heart failure, LVAD, heart transplant, institutional preparedness, infection, quarantine, protocols, collaboration 